\title{
Endometrial stromal sarcoma of the uterus with malignant thrombosis of the iliac and femoral veins: Case report and review of literature
}

\author{
Priyadarshini Tiwari ${ }^{1}$, Arvind Lele ${ }^{1}$, Rajni Vishnoi ${ }^{2}$, Nishin Jain ${ }^{3}$ \\ 1. Department of Obstetrics and Gynecology, Netaji Subhash Chandra Bose Medical College, Jabalpur (M.P.), India. 2. \\ Department of Pathology, Netaji Subhash Chandra Bose Medical College, Jabalpur (M.P.), India. 3. MP MRI and CT scan, \\ Netaji Subhash Chandra Bose Medical CollegeJ abalpur (M.P.), India.
}

Correspondence: Priyadarshini Tiwari. Address: R-2, Doctors' Colony, Medical College campus, Jabalpur, (M.P.), India, 482003. Telephone: 09-407-339-764. Email: drpriya2004@yahoo.co.in.

Received: May 13, 2012

Accepted: August 6, $2012 \quad$ Published: December 1, 2012

DOI : $10.5430 / j b g c . v 2 n 2 p 97$

URL: http://dx.doi.org/10.5430/jbgc.v2n2p97

\begin{abstract}
A case report of endometrial stromal sarcoma is presented. A 37 year old patient was admitted with a history of excessive bleeding per vaginum during menstruation and lump in abdomen for the past 6-7 months which was found to be about 24 weeks pregnancy size. On opening the abdomen for hysterectomy the uterus showed a growth extending into the left adnexa, sigmoid mesocolon, and left parametrium. During surgery there was accidental transection of the left ureter and external iliac vein which showed extensive thrombus. As total hysterectomy was not possible, subtotal hysterectomy with bilateral salpingo-oophorectomy was performed, with D-J stenting of the ureter and ligation of the external iliac vein. Postoperatively the diagnosis of thrombosis of the internal iliac, external iliac, femoral and popliteal veins was established by CT scan and Doppler imaging. Histopathology showed endometrial stromal sarcoma.
\end{abstract}

\section{Key words}

Sarcoma, Endometrial stromal, Thrombosis, Malignant

\section{I ntroduction}

Endometrial stromal sarcoma is a rare variety of malignant uterine tumor that constitutes approximately $10 \%$ of all uterine sarcomas but only about $0.2 \%$ of all uterine malignancies ${ }^{[1]}$. Though termed low grade, they have a tendency to metastasize into venous channels and have a high recurrence rates after primary surgery. Though uterine sarcomas generally occur in the post menopause, endometrial stromal sarcomas tend to have a lower median age of incidence, between 45 to 53 years ${ }^{[1]}$. Frequently, a diagnosis of uterine fibroid is made and the diagnosis of sarcoma is established only on histopathology. A high index of suspicion is required to make a preoperative diagnosis in young patients. These tumors are distinct in their tendency to form malignant thrombii into major veins, namely the iliac veins, inferior vena cava and rarely into the right atrium and ventricle. The previous distinction into low grade and high grade stromal sarcomas has fallen out of favor and the low grade variety is now named endometrial stromal sarcoma while the high grade variety is named endometrial sarcoma ${ }^{[1]}$. Surgery is the mainstay of treatment with adjuvant chemotherapy and radiation therapy offering reduced recurrence rates in some, but not all cases. 


\section{Case report}

A thirty seven year old patient was admitted to the department of obstetrics and gynecology, N.S.C.B. Medical College, Jabalpur with a lump in abdomen for the last 6 months, excessive menstrual flow for the last 5 - 6 months, and continuous bleeding per vaginum for the last 8 days.

The patient was Para 4 with three living issues. (A Para 4 is a patient who has delivered four times after the period of viability, 24 weeks of gestation). Tubal ligation had been done 10 years ago. The present menstrual cycle was 6 - 8 days/ 30 - 35 days with increased flow and passage of clots. General examination showed pallor. Per abdomen a mass of 24 weeks size pregnancy with well-defined margins could be palpated. On per speculum examination the cervix was high up and no growth was visible on the cervix and vagina. On per vaginal examination, the growth was felt to be arising from the uterus. The left parametrium showed induration and complete obliteration up to the lateral pelvic wall on both per vaginal and per rectal examination. A provisional diagnosis of impacted calcified fibroid was made.

On investigation Hemoglobin was 7.0 gm\%. The peripheral smear showed microcytic hypochromic anemia. Electrophoresis showed adult hemoglobin.

Liver function tests, renal function tests were within normal limits. (Blood Urea $22.0 \mathrm{mg} / \mathrm{dL}$, serum creatinine 0.88 mg/dl),( Total serum bilirubin $0.45 \mathrm{mg} / \mathrm{dL}$, AST $28 \mathrm{IU} / \mathrm{L}$, ALT $14 \mathrm{IU} / \mathrm{L}$ ), Prothrombin Time 16 sec: control $14 \mathrm{sec}$, Bleeding time 1minute, Clotting time $4 \mathrm{~min} 30 \mathrm{sec}$

Cervical cytology showed inflammatory cells.

USG of whole abdomen showed normal liver and spleen. Pelvic ultrasound showed multiple heterogenous lesions seen in the uterus and cervical region, the largest being $7.2 \mathrm{~cm} \times 6 \mathrm{~cm}$ suggestive of multiple uterine fibroids. On the left side there was evidence of mildly dilated pelvicalyceal system suggestive of mild hydrouretronephrosis. There was no comment on the endometrium. No evidence of lymphadenopathy was seen.

The patient did not consent to get a CT scan at this stage.

Since the patient was anemic with heavy flow during menses, a decision for hysterectomy was taken after improving hemoglobin with injectable iron.

During laparotomy the uterus was enlarged to 22 - 24 weeks size iwith irregular surface (see Figure 1). The left broad ligament and round ligament were infiltrated with the growth and the round ligament looked hypertrophied (see Figure 1). Posteriorly the uterine growth extended up to the mesentery of the sigmoid colon (see Figure 1). During surgery, the left ureter and left external iliac vein were accidentally transected as both these structures were engulfed by the growth. End to end anastomosis of the ureter was performed with DJ stenting. The left external iliac vein showed extensive malignant thrombus. There was no bleeding from the vein. As no vascular surgeon was available, the thrombus could not be removed and the vein was ligated. Due to extensive parametrial infiltration, it was not possible to free the cervix on the left and hence subtotal hysterectomy with bilateral salpingo oophorectomy was performed. No other deposits were found in the peritoneum. On questioning after surgery the patient gave a history of unilateral leg edema on the left side. Her post operative recovery was uneventful.

Postoperatively CT scan showed extensive thrombosis of external (see Figure 2) and internal iliac vein (see Figure 3) extending into the femoral vein (see Figure 4) and initial part of common iliac vein (see Figure 5). The inferior vena cava was free of thrombus (see Figure 6) Evidence of few nodular densities was seen in the left paraaortic region (see Figure 7). Venous doppler showed extensive thrombus extending from the left external Iliac vein upto the left popliteal vein. Cut section of the uterus showed ball like masses. The endometrium was $0.2 \mathrm{~cm}$ and showed one polyp which was grayish 
white. One huge mass measuring $9 \mathrm{~cm} \times 8 \mathrm{~cm} \times 7 \mathrm{~cm}$ extended into the left cornu and left ovary, bulging outside the myometrium. The left ovary was enlarged, $5 \mathrm{~cm} \times 4 \mathrm{~cm} \times 3 \mathrm{~cm}$. The left tube was swollen and the lumen was obliterated. The right adnexa were normal. Microscopy revealed endometrial stromal sarcoma showing oval small tumor cells in solid masses with dark nuclei resembling endometrial stroma arranged in masses around arterioles (see Figure 8). There was evidence of fibrosis and invasion in the stroma (see Figure 9). Lymphovascular invasion was clearly visible (see Figure 10) and also invasion of the left tube and ovary. The right tube and ovary were normal.

The diagnosis was endometrial stromal sarcoma extending into the myometrium, left tube and ovary.

The patient was referred for adjuvant chemotherapy and radiation therapy after postoperative recovery.

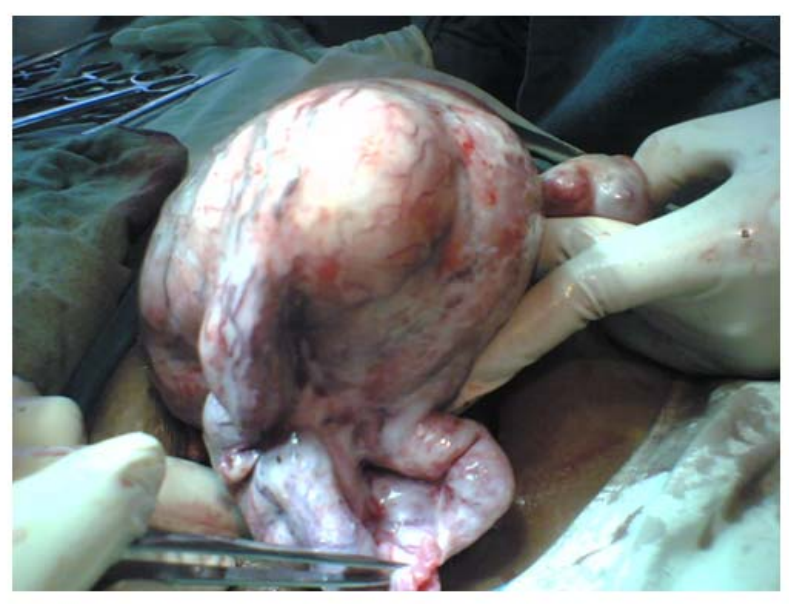

Figure 1. Operative photograph showing enlarged uterus and extension into the left adnexa

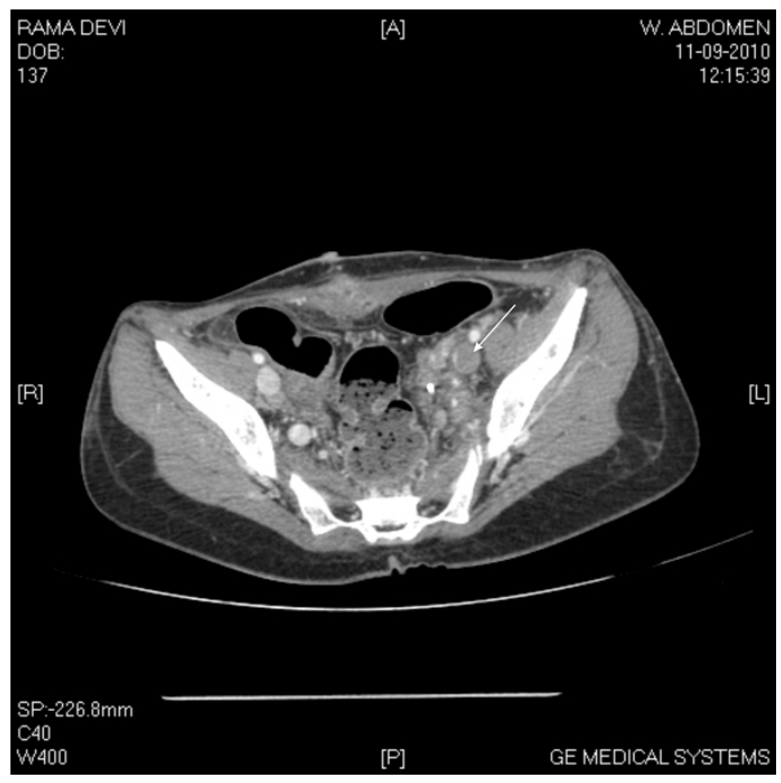

Figure 2. CT scan showing thrombus in external iliac vein 


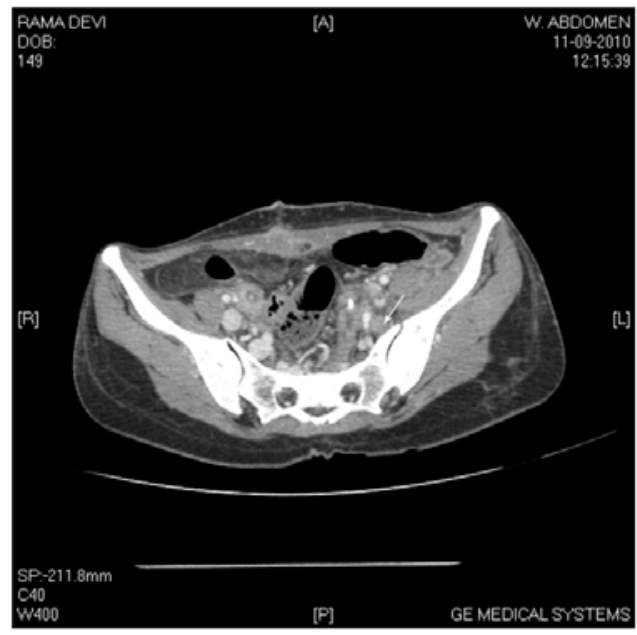

Figure 3. CT scan showing thrombus in internal iliac vein

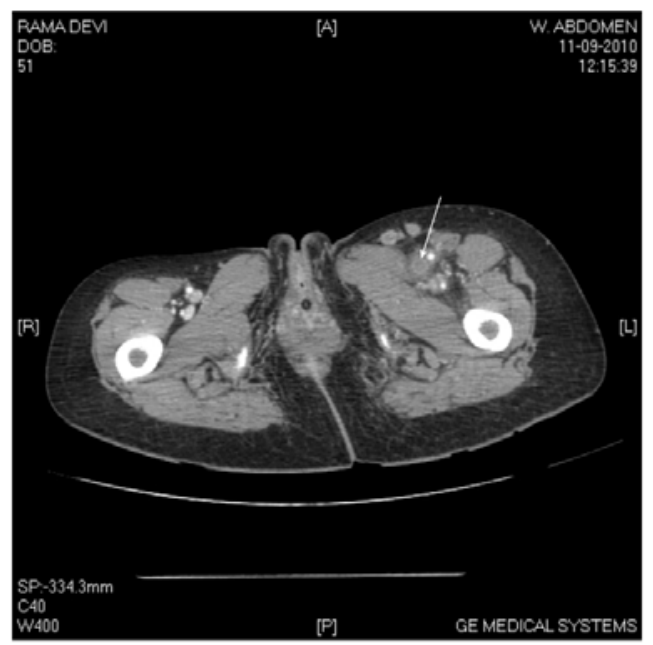

Figure 4. CT scan showing thrombus in femoral vein

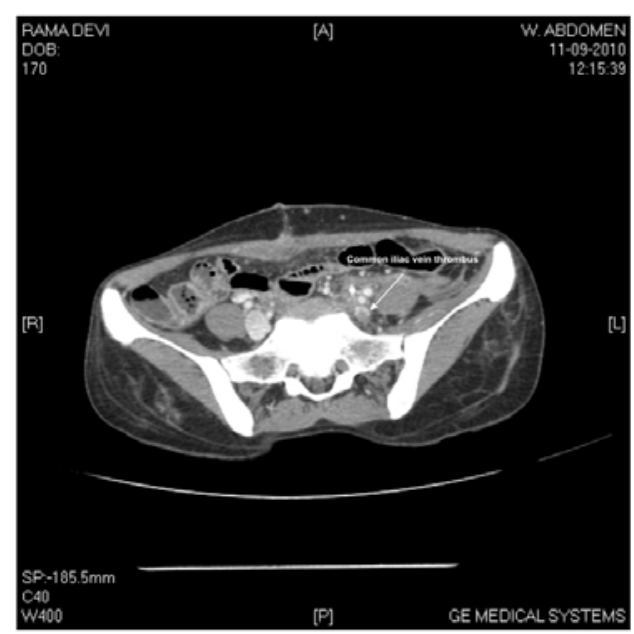

Figure 5. CT scan showing thrombus in initial part of common iliac vein 


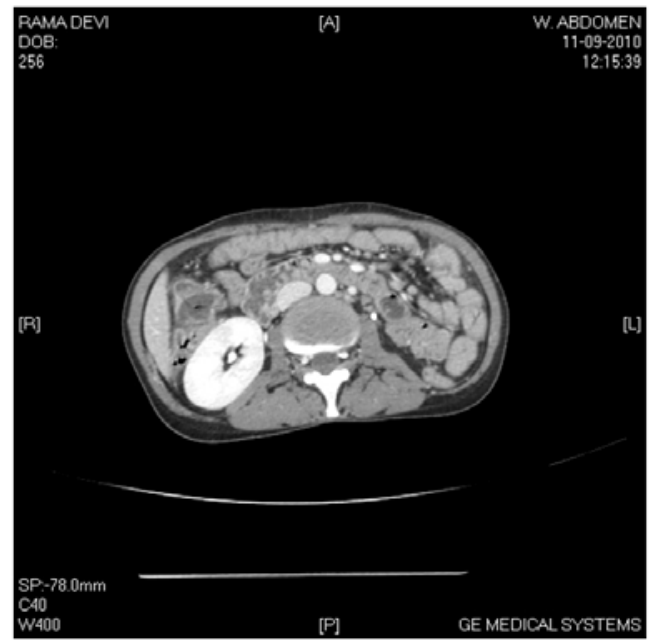

Figure 6. CT scan showing inferior vena cava free of thrombus

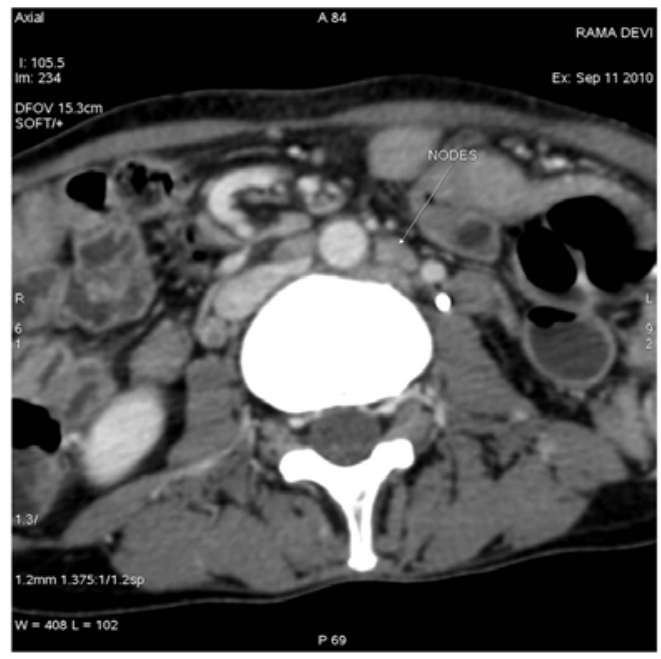

Figure 7. CT scan showing nodular densities in the left para aortic region

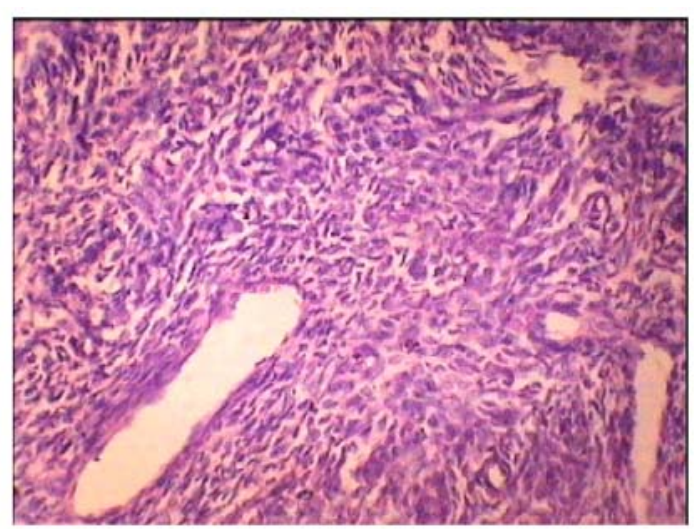

Figure 8. Histology of Endometrial Stromal Sarcoma 


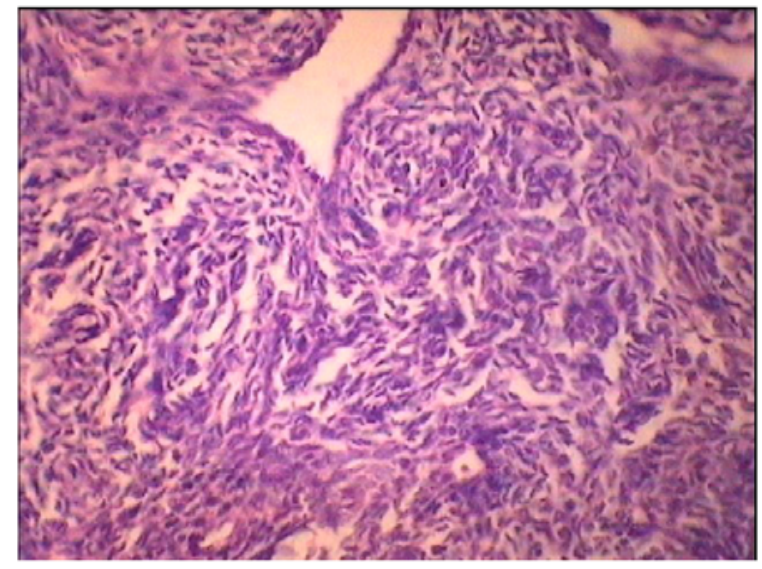

Figure 9. Histology showing fibrosis and stromal invasion

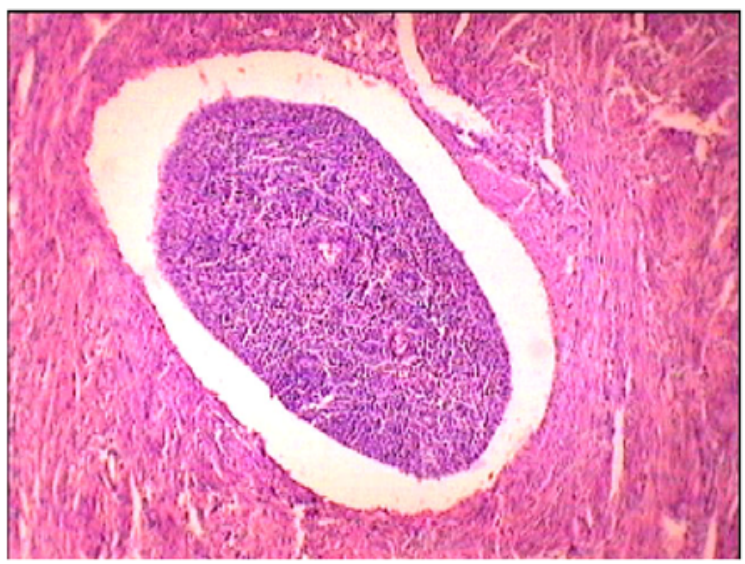

Figure 10. Histology showing lympho vascular invasion

\section{Discussion and review of literature}

Endometrial stromal sarcoma is a rare variety of malignant uterine tumor forming about $10 \%$ of all sarcomas and $0.2 \%$ of all uterine malignancies ${ }^{[1]}$. Distinction between low grade and high grade Endometrial Stromal Sarcoma is based on the number of mitotic figures ${ }^{[1]}$. This classification has now fallen out of favor and now the same entities are classified as endometrial stromal sarcoma and endometrial sarcoma respectively.

Most often sarcomas affect postmenopausal women ${ }^{[1]}$. The median age for endometrial stromal sarcomas is lower, $44 \pm$ $6 y r s$. Generally these patients do not have the risk factors for endometrial cancer. They may present with abnormal vaginal bleeding, progressive menorrhagia and abdominal pain. ESS can be mistaken for leiomyoma. Its clinical recognition may be difficult, and the diagnosis is often made postoperatively after histological examination ${ }^{[1]}$. Rapid enlargement of a fibroid may point towards the diagnosis.

This is a rare kind of angioinvasive tumor with a high recurrence rate of about $50 \%$ after a long period of latency ${ }^{[2]}$. Despite the high recurrence rates these tumors have a good prognosis ${ }^{[3]}$. 
Tumor extension has been reported into iliac veins, femoral veins, inferior vena cava, right atrium, rarely going into the right ventricle ${ }^{[2,4-6]}$ and pulmonary artery ${ }^{[7]}$. Invasion into the infrarenal part of the aorta has also been reported ${ }^{[2]}$. Despite its good prognostic nature ESS may behave as an aggressive malignancy ${ }^{[4]}$.

Primary treatment generally consists of total abdominal hysterectomy with bilateral salpingooophorectomy.

Extensive radical surgery can be performed safely in case of venous thrombus and improves disease free survival ${ }^{[2]}$. Complete primary surgery offers long disease free survival ${ }^{[8]}$ but nearly $50 \%$ will recur ${ }^{[1]}$. Tumor thrombectomy has been reported without bypass ${ }^{[9]}$ and with hypothermic circulatory arrest ${ }^{[10]}$.

Many clinicians advocate use of adjuvant chemotherapy, radiation therapy and/or hormone therapy to suppress tumor recurrences ${ }^{[1]}$.

An excellent response has been obtained on adjuvant treatment with megestrol and leuprolide ${ }^{[3]}$.

\section{Acknowledgements}

The authors wish to thank Dr. Neeta Bhatia, Senior Consultant, National Hospital, Jabalpur, M.P. for her help with the photographs of histopathology slides.

\section{References}

[1] Tahereh Ashraf-Ganjoei , Nadereh Behtash, Mamak Shariat and Asamosadat Mosavi. Low grade Endometrial Stromal Sarcoma of uterine corpus, a clinico-pathological and survey study in 14 cases. World Journal of Surgical Oncology. 2006 ; 4 : 50. PMid:16895611 http://dx.doi.org/10.1186/1477-7819-4-50.

[2] Renzulli P, Weimann R, Barras JP, Carrel TP, Candinas D. Low-grade endometrial stromal sarcoma with inferior vena cava tumor thrombus and intracardiac extension: radical resection may improve recurrence free survival. Surg Oncol. 2009 Mar; 18(1): 57-64. Epub 2008 Aug 15. PMid:18708288 http://dx.doi.org/10.1016/j.suronc.2008.07.003

[3] Dupont NC, Disaia PJ. Recurrent endometrial stromal sarcoma: treatment with a progestin and gonadotropin releasing hormone agonist. Sarcoma. 2010; 2010: 353679. Epub 2010 Jun 10

[4] Gabal S, Ashour Z, Hamada G, Aziz SA, Khairy H, Badawy H, Hamada EM, Saied K. Low-grade endometrial stromal sarcoma with intravenous extension to the heart. Medscape J Med. 2009; 11(1): 23. Epub 2009 Jan 23. PMid:19295944

[5] Yokoyama Y, Ono Y, Sakamoto T, Fukuda I, Mizunuma H. Asymptomatic intracardiac metastasis from a low-grade endometrial stromal sarcoma with successful surgical resection. Gynecol Oncol. 2004 Mar; 92(3): 999-1001. PMid:14984976 http://dx.doi.org/10.1016/j.ygyno.2003.11.049

[6] Suzuki S, Yasuhara K, Koyano T, Obayashi T. Tumor thrombectomy for endometrial stromal sarcoma extending into the inferior vena cava and the right atrium from the uterus]. Kyobu Geka. 2008 Feb; 61(2): 139-42. PMid:18268952

[7] Fernando Val-Bernal J, Hernández-Nieto E.Symptomatic intracavitary (noninvasive) cardiac metastasis from low grade endometrial stromal sarcoma of the uterus. Pathol Res Pract. 1999; 195(10): 717-22. http://dx.doi.org/10.1016/S0344-0338(99)80067-3

[8] Veroux P, Veroux M, Nicosia A, Bonanno MG, Tumminelli MG, Milone P, Petrillo G. Thrombectomy of the inferior vena cava from recurrent low-grade endometrial stromal sarcoma: case report and review of the literature. J Surg Oncol. 2000 May; 74(1): 45-8. http://dx.doi.org/10.1002/1096-9098(200005)74:1<45::AID-JSO11>3.0.CO;2-K

[9] Jibiki M, Inoue Y, Sugano N, Iwai T, Katou T. Tumor thrombectomy without bypass for low-grade malignant tumors extending into the inferior vena cava: report of two cases. Surg Today. 2006; 36(5): 465-9. PMid:16633754 http://dx.doi.org/10.1007/s00595-005-3175-4

[10] Phillips MR, Bower TC, Orszulak TA, Hartmann LC. Intracardiac extension of an intracaval sarcoma of endometrial origin. Ann Thorac Surg. 1995 Mar; 59(3): 742-4. http://dx.doi.org/10.1016/0003-4975(94)00580-X 